\title{
Evaluation of T2Candida Panel for Detection of Candida in Peritoneal
}

\section{Dialysates}

Running Title: T2Candida use in peritoneal dialysate

Anne M. Kouri, MD ${ }^{\mathrm{a}}$; Theodore W. Kieffer, MD, MS ${ }^{\mathrm{b}}$; Corina Nailescu, MD ${ }^{\mathrm{a}}$; Jeffrey Leiser, MD, $\mathrm{PhD}^{\mathrm{a}}$; Bryan H. Schmitt, $\mathrm{DO}^{\mathrm{c}}$; Ryan F. Relich, $\mathrm{PhD}^{\mathrm{c}}$; Thomas E. Davis, $\mathrm{MD}^{\mathrm{c}}$, PhD; John J. Manaloor, $\mathrm{MD}^{\mathrm{d}}$

Indiana University School of Medicine

Indianapolis, IN

a Department of Pediatric Nephrology and Hypertension; ${ }^{c}$ Department of Pathology and

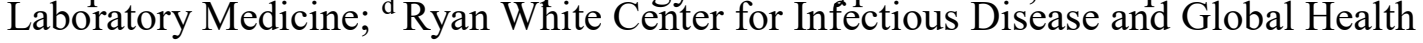

${ }^{\mathrm{b}}$ West Virginia School of Medicine

Morgantown, WV

Department of Pathology, Anatomy, and Laboratory Medicine

Acknowledgements:

We thank the dialysis nursing staff at Riley Hospital for Children at Indiana University Health for their assistance in the anonymous collection of the dialysates and the Indiana University Health Division of Clinical Microbiology for use of their T2Biosystems instrumentation. T2Biosystems supplied the testing kits for this study. T2Biosystems did not have involvement in data collection, analysis or interpretation or in the writing of this manuscript. They did approve of submitting this for publication.

Corresponding Author:

Anne M. Kouri, MD

699 Riley Hospital Drive, Rm 230

Indianapolis, IN 46202

Phone: (317)-274-2563

Fax: (317)-278-3599

akouri@iupui.edu

This manuscript contains 1196 words, 1 figure, 1 table.

This is the author's manuscript of the article published in final edited form as:

Kouri, A.M., Kieffer, T.W., Nailescu, C., Leiser, J., Schmitt, B.H., Relich, R.F., Davis, T.E., Manaloor, J.J. (2019).

Evaluation of T2Candida Panel for Detection of Candida in Peritoneal Dialysates. Peritoneal Dialysis International, 40(1), pp 96-99. https://doi.org/10.1177/0896860819879874 


\begin{abstract}
Fungal peritonitis in the peritoneal dialysis population is difficult to diagnose promptly due to the inherently slow cultivation-based methods currently required for identification of peritonitis pathogens. Because of the moderate risk for severe complications, the need for rapid diagnostics is considerable. One possible solution to this unmet need is the T2Candida Panel, a new technology designed to detect the most common pathogenic Candida spp. directly from whole blood specimens in as little as a few hours. We hypothesized that this technology could be applied to the detection of Candida in peritoneal dialysate, a matrix not currently approved by the Food and Drug Administration for testing by this system. Remnant dialysate samples from three healthy (noninfected) pediatric peritoneal dialysis patients were spiked with Candida glabrata, serially diluted, and tested in triplicate with unaltered dialysate specimens. The assay detected C. glabrata in 100\% of spiked dialysate samples across the full spectrum of dilutions tested, and no assay inhibition or cross-reactivity was noted. These findings suggest one of possibly more applications of this technology. The positive clinical implications of this test will continue to be realized as its use is validated in peritoneal dialysate and other patient specimen types.
\end{abstract}




\section{KEYWORDS}

Peritonitis

Fungal peritonitis

T2Candida

Candida peritonitis

T2Dx

\section{INTRODUCTION}

Peritoneal dialysis is often the preferred form of renal replacement therapy for pediatric patients with end-stage renal disease (ESRD) (1). One complication of peritoneal dialysis is severe and potentially life-threatening peritonitis $(1,2)$.

Fungal peritonitis in the peritoneal dialysis population is rare; however, it is associated with high morbidity and mortality $(2,3)$. The most commonly implicated fungal pathogens in peritonitis include Candida albicans, Candida parapsilosis, and Candida glabrata (3). The management of fungal peritonitis is unique because prompt removal of the peritoneal dialysis catheter is required in addition to initiation of antifungal treatment (4). Currently, the gold standard for the diagnosis of fungal peritonitis is fungal culture, a slow process that can take up to 24-48 hours, resulting in significant diagnostic and therapeutic delays.

A novel magnetic resonance-based technology has emerged for the detection of the most common causes of candidemia, including C. albicans, C. glabrata, C. parapsilosis, C. tropicalis, and C. krusei. The T2Dx system (T2 Biosystems, Lexington, MA) is comprised of the T2Dx Instrument and a U.S. Food and Drug Administration (FDA)-cleared candidemia detection panel, the T2Candida Panel. This technology uses T2 magnetic resonance to detect as little as one to three 
colony forming units per milliliter $\left(\mathrm{CFU} \mathrm{m} l^{-1}\right)$ of the aforementioned Candida spp (5). Another attractive feature of this system is that it tests EDTA-anticoagulated whole blood specimens, obviating the need for cultivation prior to sample analysis (6). When used in the appropriate context, the T2 system has been shown to reduce the time-to-detection of candidemia to approximately 3 hours (5).

We hypothesized that that the high sensitivity of this system coupled with its rapid detection time would make it an attractive candidate for testing additional sample matrices, including peritoneal dialysate. As such, this tool could help improve patient survival, lessen the effects of fungal peritonitis on the peritoneal membrane, and curb the use of unwarranted antimicrobial therapy $(4,7,8)$. To that end, we challenged the ability of the T2Candida Panel to detect C. glabrata in samples of peritoneal dialysate. 


\section{METHODS}

Ethics statement. This study was approved by the IRB of the IU School of Medicine, Indianapolis, IN. All samples in this study were de-identified to protect the privacy of the patients. Informed consent was not required.

There were three main steps to conducting this study: (1) Retrieving dialysate samples from otherwise healthy (non-infected) patients; (2) Spiking the samples with C. glabrata in the laboratory; (3) Analyzing the specimens on with the T2Candida Panel. See Figure 1.

Dialysate fluid procurement and testing. Samples were collected from peritoneal dialysis patients randomly during their monthly routine clinic visit. They brought their dialysate from home for their routine $\mathrm{Kt} / \mathrm{V}$ calculation. Two $60-\mathrm{mL}$ syringes of this remnant dialysate from each patient were submitted for experimentation. Remnant peritoneal dialysates from three pediatric patients were used for all analyses described herein. Dialysate compositions of $1.5 \%$ and $2.5 \%$ dextrose, and a combination of $1.5 \%$ and $2.5 \%$ dextrose, were selected for $\mathrm{T} 2$ analysis and manipulation to assess for interference over a range of dextrose concentrations.

Preparation of contrived dialysates. Prior to experimental manipulation, dialysates were stored at $4^{\circ} \mathrm{C}$. All dialysate specimens were spiked and analyzed by the T2Dx instrument within $48 \mathrm{~h}$ of sample collection (range, 11:15 h-41:44 h; mean, 29:09 h). Suspensions of Candida glabrata ATCC $^{\circledR}$ MYA-2950 ${ }^{\mathrm{TM}}$ were prepared using the remnant peritoneal dialysate fluid described above. Briefly, C. glabrata was grown on Sabouraud dextrose agar (SDA) and colonies were transferred to sterile normal saline aliquots. The turbidity of each suspension was adjusted to the approximate 
density of a 0.5 McFarland standard. $10^{-2}, 10^{-4}$ and $10^{-6}$ dilutions, prepared using dialysate, of 0.5 MF C. glabrata solution were subsequently created for T2Candida Panel analysis. C. glabrata concentrations were confirmed by colony counts from $0.1 \mathrm{~mL}$ of dialysate solutions incubated on SDA plates.

T2Candida Panel analysis. Briefly, $10^{-2}\left(3 \times 10^{4} \mathrm{CFU} / \mathrm{mL}\right), 10^{-4}(300 \mathrm{CFU} / \mathrm{mL})$, and $10^{-6}$ (3CFU/mL) standard dilutions, each in triplicate, of C. glabrata-spiked dialysate were transferred to 4-ml BD Vacutainer ${ }^{\circledR}$ tubes (BD, Franklin Lakes, NJ) containing EDTA and were tested using the T2Candida Panel according to the manufacturer's instructions for whole blood analysis. Undiluted, non-spiked dialysate fluids were tested concurrently as negative controls. The time from standard dilution preparation to automated analysis was less than one hour. 


\section{RESULTS}

Assay results were released from the T2Dx system qualitatively in three categories: $C$.

albicans/C. tropicalis, C. parapsilosis, and C. glabrata/C. krusei. All assay results were valid with no interference identified. There was $100 \%$ concordance between spiked specimens and negative controls with test results, and therefore the expected results were obtained. See Table 1 for complete assay results including glucose concentrations of dialysates.. 


\section{DISCUSSION}

To our knowledge, this is the first study of its kind. Although limited to three dialysate samples, we reproducibly demonstrated that the T2Candida Panel was able to detect C. glabrata in spiked peritoneal dialysate samples. However, the results in this study should be considered preliminary.

C. glabrata alone was chosen for this pilot study due to a limited number of testing kits and uniqueness among Candida species for not producing pseudohyphae, which made organism quantification more accurate. We also chose to use remnant dialysate to simulate, as best as possible, the sample matrices that would be obtained directly from patients with fungal peritonitis.

This test has the potential to have profound clinical relevance in patients with suspected fungal peritonitis. Early detection of fungal pathogens allowing for prompt treatment may improve patient survival. Other potential benefits include narrowing of antimicrobial coverage to mitigate toxicity and untoward side effects as well as prevent the spread of antimicrobial resistance. For instance, intraperitoneal cefepime administration has been rarely associated with neurotoxicity and IP vancomycin has been associated with significant antimicrobial resistance (4). Thus, patients with atypical or severe presentations of peritonitis could benefit from early detection of a causative organism to improve morbidity and mortality the infection.

ACKNOWLEDGEMENTS: We thank the dialysis nursing staff for their assistance in the collection of the dialysates and the IUH Division of Clinical Microbiology for use of T2Biosystems instrumentation. T2Biosystems supplied the testing kits for this study. T2Biosystems did not have involvement in data collection, analysis or interpretation or in the writing of this manuscript. They did approve of submitting this for publication. 


\section{CONFLICT OF INTEREST DISCLOSURE}

We have read and understood Peritoneal Dialysis International's policy on conflicts of interest disclosure and declare that we have none.

\section{HUMAN AND ANIMAL RIGHTS}

Ethical approval: All procedures performed in studies involving human participants were in accordance with the ethical standards of the institutional and/or national research committee at which the studies were conducted (IRB approval number 1606331472R002) and with the Helsinki Declaration of 1975 and its later amendments or comparable ethical standards. 


\section{REFERENCES}

1. Woodrow G, Fan SL, Reid C, Denning J, Pyrah AN. Renal Association Clinical Practice Guideline on peritoneal dialysis in adults and children. Bmc Nephrol. 2017;18.

2. Auricchio S, Giovenzana ME, Pozzi M, Galassi A, Santorelli G, Dozio B, et al. Fungal peritonitis in peritoneal dialysis: a 34-year single centre evaluation. Clin Kidney J. 2018;11(6):874-80.

3. Matuszkiewicz-Rowinska J. Update on fungal peritonitis and its treatment. Perit Dial Int. 2009;29 Suppl 2:S161-5.

4. Warady BA, Bakkaloglu S, Newland J, Cantwell M, Verrina E, Neu A, et al. Consensus guidelines for the prevention and treatment of catheter-related infections and peritonitis in pediatric patients receiving peritoneal dialysis: 2012 update. Perit Dial Int. 2012;32 Suppl 2:S3286.

5. Neely LA, Audeh M, Phung NA, Min M, Suchocki A, Plourde D, et al. T2 magnetic resonance enables nanoparticle-mediated rapid detection of candidemia in whole blood. Sci Transl Med. 2013;5(182):182ra54.

6. T2Biosystems. Evaluation of Automatic Class III Designation for T2Candida Panel and T2Dx Instrument: Decision Summary. January 12, 2015 ed2015.

7. Hamula CL, Hughes K, Fisher BT, Zaoutis TE, Singh IR, Velegraki A. T2Candida Provides Rapid and Accurate Species Identification in Pediatric Cases of Candidemia. Am J Clin Pathol. 2016;145(6):858-61.

8. Beyda ND, Alam MJ, Garey KW. Comparison of the T2Dx instrument with T2Candida assay and automated blood culture in the detection of Candida species using seeded blood samples. Diagn Microbiol Infect Dis. 2013;77(4):324-6. 
Running Title: T2Candida use in dialysate fluid

\section{LEGEND}

Figure 1: Study Protocol 


\begin{tabular}{|c|c|c|c|c|c|c|c|}
\hline \multirow{2}{*}{$\begin{array}{c}\text { Dialysate } \\
\text { dextrose } \\
\text { concentration }\end{array}$} & \multicolumn{2}{|c|}{$\begin{array}{l}\text { Actual measured } \\
\text { glucose (mg/dL) }\end{array}$} & \multirow{2}{*}{ 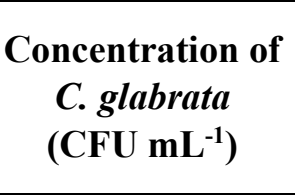 } & \multirow{2}{*}{$\begin{array}{l}\text { Number of } \\
\text { specimens }\end{array}$} & \multirow{2}{*}{$\begin{array}{c}\text { C. albicans/C. } \\
\text { tropicalis }\end{array}$} & \multirow{2}{*}{ C. parapsilosis } & \multirow{2}{*}{$\begin{array}{r}\text { C. glabrata/ } \\
\text { C. krusei0 }\end{array}$} \\
\hline & $\begin{array}{c}\text { Syringe } \\
1\end{array}$ & $\begin{array}{c}\text { Syringe } \\
2\end{array}$ & & & & & \\
\hline \multirow{4}{*}{$1.5 \%$} & \multirow{4}{*}{1050} & \multirow{4}{*}{1059} & $>1000$ & 3 & Negative (3) & Negative (3) & Positive (3) \\
\hline & & & 380 & 3 & Negative (3) & Negative (3) & Positive (3) \\
\hline & & & 3.3 & 3 & Negative (3) & Negative (3) & Positive (3) \\
\hline & & & 0 (neg ctrl) & 2 & Negative (2) & Negative (2) & Negative (2) \\
\hline \multirow{4}{*}{$1.5 \%+2.5 \%$} & \multirow{4}{*}{1120} & \multirow{4}{*}{1129} & $>1000$ & 3 & Negative (3) & Negative (3) & Positive (3) \\
\hline & & & 470 & 3 & Negative (3) & Negative (3) & Positive (3) \\
\hline & & & 3.3 & 3 & Negative (3) & Negative (3) & Positive (3) \\
\hline & & & 0 (neg ctrl) & 2 & Negative (2) & Negative (2) & Negative (2) \\
\hline \multirow{4}{*}{$2.5 \%$} & \multirow{4}{*}{1757} & \multirow{4}{*}{1744} & $>1000$ & 3 & Negative (3) & Negative (3) & Positive (3) \\
\hline & & & 453 & 3 & Negative (3) & Negative (3) & Positive (3) \\
\hline & & & 4.4 & 3 & Negative (3) & Negative (3) & Positive (3) \\
\hline & & & 0 (neg ctrl) & 2 & Negative (2) & Negative (2) & Negative $(2)$ \\
\hline
\end{tabular}

TABLE 1 T2Candida Dialysate Assay Results 


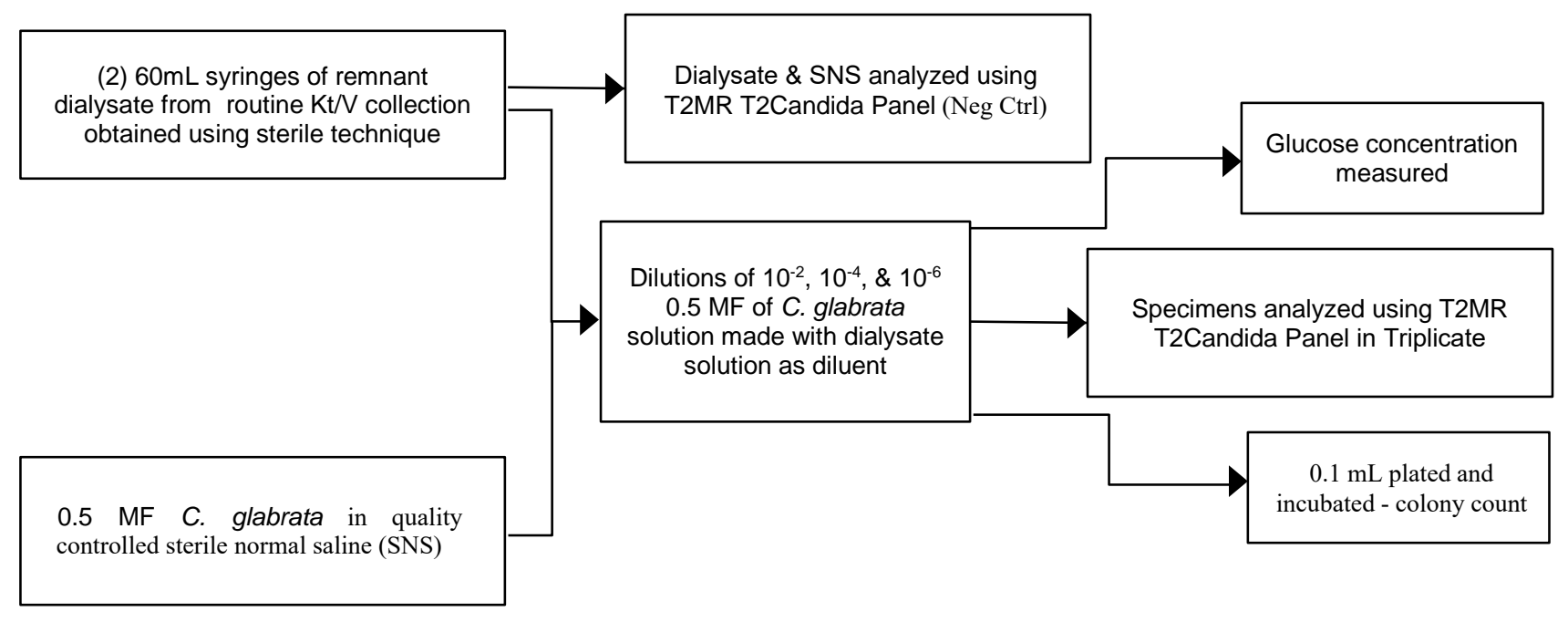

